\title{
The effects of temporary intraocular pressure spikes after intravitreal dexamethasone implantation on the retinal nerve fiber layer
}

This article was published in the following Dove Press journal: Clinical Ophthalmology

\author{
Kendall W Wannamaker \\ Sarah Kenny' \\ Rishi Das' \\ Aaron Mendlovitz ${ }^{2}$ \\ Jordan M Comstock' \\ Edward R Chu' \\ Sepehr Bahadorani' \\ Nathan J Gresores ${ }^{2}$ \\ Kinley D Beck' \\ Chelsey J Krambeer ${ }^{2}$ \\ Daniel S Kermany ${ }^{2}$ \\ Roberto Diaz-Rohena \\ Daniel P Nolan ${ }^{2}$ \\ Jeong-Hyeon Sohn' \\ Michael A Singer ${ }^{2}$ \\ 'Ophthalmology, University of Texas \\ Health Science Center San Antonio, San \\ Antonio, TX, USA; ${ }^{2}$ Medical Center \\ Ophthalmology Associates, San Antonio, \\ TX, USA
}

\begin{abstract}
Background and objective: The dexamethasone (DEX) implant is known to cause temporary intraocular pressure (IOP) spikes after implantation. The purpose of this study is to determine if IOP spikes after DEX implant cause significant thinning in the retinal nerve fiber layer (RNFL).
\end{abstract}

Study design, patients, and methods: A total of 306 charts were reviewed with 48 and 21 patients meeting inclusion criteria for the cross-sectional and prospective groups, respectively. Cross-sectional inclusion criteria: IOP spike $\geq 22 \mathrm{mmHg}$ up to 16 weeks after DEX implant, DEX implant in only 1 eye per patient, and spectral-domain optical coherence tomography (OCT) RNFL imaging of both eyes $\geq 3$ months after IOP spike. Prospective inclusion criteria: OCT RNFL performed within 1 year prior to DEX implantation, IOP spike $\geq 22 \mathrm{mmHg}$ up to 16 weeks after DEX implant, and OCT RNFL performed $\geq 3$ months after IOP spike. The average RNFL thickness in the contralateral eye was used as the control in the cross-sectional group. Institutional review board approval was obtained.

Results: In the cross-sectional group, there was no statistically significant difference in the mean RNFL thicknesses in the treated vs untreated eyes $(80.4 \pm 15.5 \mu \mathrm{m}$ and $82.6 \pm 15.8 \mu \mathrm{m}$, respectively; $P=0.33$ ) regardless of treatment diagnosis, magnitude of IOP spike, or history of glaucoma. In the prospective group, mean RNFL thicknesses before and after IOP spikes $\geq 22 \mathrm{mmHg}$ were similar $(78.0 \pm 14.8 \mu \mathrm{m}$ and $75.6 \pm 13.6 \mu \mathrm{m}$, respectively; $P=0.13$ ).

Conclusion and relevance: Temporary elevation of IOP after DEX implantation when treated with topical IOP lowering drops does not appear to lead to a meaningful change in RNFL thickness

Keywords: Ozurdex ${ }^{\circledR}$, dexamethasone, glaucoma, intraocular pressure, retinal nerve fiber layer, ocular hypertension

\section{Background and objective}

Intravitreal steroid injections have been shown to be an effective means by which to treat macular edema secondary to a wide array of etiologies including diabetes, retinal vein occlusion (RVO), cystoid macular edema (CME), wet age-related macular degeneration (wAMD), and noninfectious posterior uveitis. ${ }^{1}$ The efficacy of corticosteroids in the treatment of macular edema is linked to their known anti-inflammatory, antiedematous, and anti-angiogenic properties. $^{2-4}$ The dexamethasone (DEX) implant (0.7 or $0.35 \mathrm{mg}$ ), also known as Ozurdex ${ }^{\circledR}$, consists of micronized dexamethasone in a biodegradable copolymer which slowly releases steroids into the vitreous over a period of about 6 months (Figure 1). ${ }^{5,6}$ This sustained-release intravitreal steroid
Correspondence: Michael A Singer Medical Center Ophthalmology Associates, 9157 Huebner Road, San Antonio, TX 78240, USA

Tel + I 2102693754

Email msingerlI@me.com 

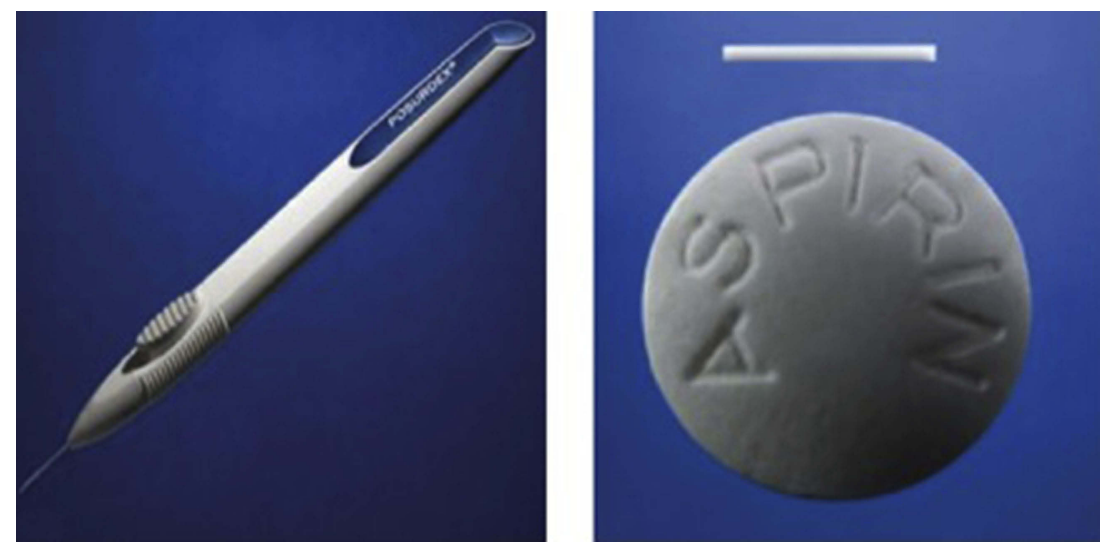

Figure I Ozurdex ${ }^{\circledR}$ injector (left) and pellet (right).

implant is FDA approved to treat macular edema associated with RVO, diabetic macular edema (DME), and noninfectious posterior uveitis and has been shown to effectively reduce macular edema leading to significantly improved visual acuity and a reduced number of anti-vascular endothelial growth factor (VEGF) injections. ${ }^{7-10}$

Despite the promising therapeutic benefit of DEX implant in the treatment of macular edema, studies have described temporary intraocular pressure (IOP) spikes as a common adverse event in up to $27-32 \%$ of the patients. ${ }^{11,12}$ Increased IOP, experimentally induced in animal models, have demonstrated similar ultrastructure retinal ganglion cell changes as seen in optic nerve fibers in postmortem human eyes that have glaucoma. ${ }^{13}$ Although IOP elevation is considered a risk factor for optic nerve damage and is the only modifiable risk factor for glaucoma, the exact pathogenesis of glaucoma is not fully understood and is likely multifactorial. ${ }^{14}$ According to a recent systematic review by Bucolo et al, the vast majority of steroid responders who receive DEX implant can be managed with topical IOP reducing drops and rarely require incisional glaucoma surgeries. ${ }^{15}$ Nevertheless, DEX implant must be used with caution and IOP checks must be performed diligently especially in patients with ocular histories of glaucoma. Whether optic nerve damage is a result of DEX-related IOP spikes or the natural progression of the vitreoretinal disease remains unclear. The purpose of this retrospective study is to determine if those patients who experienced IOP spikes following DEX implantation ultimately develop significant optic nerve damage.

\section{Study design/patients and methods}

This retrospective chart review was performed with approval from the Specialty Surgery Center Institutional Review Board affiliated with the Medical Center
Ophthalmology Associates from September 2009 to June 2018. Patient consent was not required given the retrospective nature of this study. Furthermore, the information collected had no effect on patient treatment or care. Patient identity was masked using a medical record number. All stages of this study were conducted in accordance with the principles set forth by the Declaration of Helsinki.

We reviewed 306 patient charts of patients who received DEX implants for diagnoses such as branch retinal vein occlusion (BRVO), central retinal vein occlusion (CRVO), DME, noninfectious posterior uveitis, wAMD, and CME with 48 and 21 patients meeting inclusion criteria for the cross-sectional and prospective groups, respectively. Inclusion criterion for the cross-sectional group consisted of IOP spikes $\geq 22 \mathrm{mmHg}$ up to 16 weeks after DEX implant, DEX implant in only 1 eye per patient, and optical coherence tomography (OCT) RNFL imaging of both eyes per patient performed $\geq 3$ months after the IOP spike. Inclusion criteria for the prospective group consisted of OCT RNFL imaging performed within 1 year prior to DEX implantation, IOP spike $\geq 22 \mathrm{mmHg}$ up to 16 weeks after DEX implant, and OCT RNFL imaging performed $\geq 3$ months after the IOP spike. Use of IOP lowering drops, prior anti-VEGF treatment, and past ocular history of glaucoma were not exclusion criterion. Patients were excluded for any prior history of optic neuropathy, except for glaucoma (eg, ischemia, trauma, tumor). Informed consent was obtained for all patients prior to intravitreal injections.

The included patients underwent a complete ophthalmological examination prior to DEX implantation. Demographic data, past ocular history of glaucoma, and central corneal thickness (CCT) were recorded. IOP was noted before DEX implantation, at the time of the IOP spike, and at 4-8 weeks after the IOP spike. IOP was 
measured using a Tonopen. The cross-sectional patients were stratified based on the magnitude of IOP elevation after DEX implantation as follows: IOP $22-24 \mathrm{mmHg}$, $25-29 \mathrm{mmHg}, \geq 30 \mathrm{mmHg}$, and $\geq 10 \mathrm{mmHg}$ from baseline IOP. In addition, these patients were stratified by diagnoses including BRVO, DME, CRVO, and other (posterior uveitis, wAMD, CME), as well as a past ocular history of glaucoma. Average circumpapillary RNFL thickness measured by the Zeiss Cirrus spectral-domain OCT was used to quantify changes in the RNFL thickness. OCT signal strength was recorded and compared between treatment and control arms. The number of IOP lowering drops used to treat IOP spikes were also obtained. The average RNFL thickness in the contralateral eye was used as the control in the cross-sectional group. All data are presented as mean \pm standard deviation (SD). An unpaired $t$-test was used to determine statistical significance between mean values (eg, average RNFL) comparing treatment and control arms in the cross-sectional group. A paired $t$-test was used to determine statistical significance between mean RNFL values before (control) and after the IOP spike in the prospective group. A $P$-value of $<0.05$ was considered the threshold for significance.

\section{Results}

\section{Cross-sectional group}

We included 96 eyes from 48 patients with the number of eyes treated and untreated split into 2 groups evenly. Demographic data for patients in this group are listed in Table 1 . As high as $48 \%$ of the patients had a past ocular history of glaucoma. The mean time between initial IOP spike and OCT imaging was 18 months ranging from 4 to 83 months (median of 13.5 months). Mean OCT signal strength was not significantly different between treatment and control arms $(6.3 \pm 1.7$ and $6.5 \pm 1.5$, respectively; $P=0.45$ ). The mean number of DEX implants before the IOP spike was 2.1 (range: 1-12). Treatment and control arms had similar IOPs before DEX implant $(15.4 \pm 3.4$ $\mathrm{mmHg}$ and $15.1 \pm 2.7 \mathrm{mmHg}$, respectively; $P=0.64$ ). The average IOP of the treated eye at the time of the IOP spike was $26.4 \pm 4.3 \mathrm{mmHg}$ in contrast to the average IOP of the untreated eye during the same time at $16.5 \pm 3.6 \mathrm{mmHg}$ $(P<0.01)$. The average IOP elevation stratified by diagnosis is demonstrated in Figure 2. IOP remained significantly higher in the treated eye compared to the control eye at 4-8 weeks after the IOP spike, although to a lesser degree (17.0 $\pm 3.9 \mathrm{mmHg}$ and $15.2 \pm 3.4 \mathrm{mmHg}$, respectively; $P=0.02$ ).
Table I Demographic data

\begin{tabular}{|l|l|l|}
\hline \multicolumn{2}{|l|}{ Demographics (n=48 patients - 96 eyes) } \\
\hline Age (yrs) & & $72.7 \pm 10.9$ \\
& Male & $51 \%$ \\
& Female & $49 \%$ \\
\hline Race & & \\
& White & $57 \%$ \\
& Hispanic & $29 \%$ \\
& Black & $1 \%$ \\
& Other & $13 \%$ \\
\hline \multirow{2}{*}{ Diagnosis } & & \\
& BRVO & $34 \%$ \\
& DME & $25 \%$ \\
& CRVO & $23 \%$ \\
& Uveitis & $6 \%$ \\
& CME & $6 \%$ \\
& WAMD & $4 \%$ \\
\hline
\end{tabular}

Abbreviations: BRVO, branch retinal vein occlusion; DME, diabetic macular edema; CRVO, central retinal vein occlusion; WAMD, wet age-related macular degeneration; CME, cystoid macular edema.

When comparing the IOP of the treated eye at the time of the IOP spike and 4-8 weeks later, there was a significant decreased in IOP $(26.4 \pm 4.3 \mathrm{mmHg}$ and $17.0 \pm 3.9 \mathrm{mmHg}$, respectively; $P<0.01$ ). Mean CCT was not significantly different between treated $(569 \mu \mathrm{m})$ and untreated $(571 \mu \mathrm{m})$ eyes $(P=0.61)$. As high as $76 \%$ of the patients with IOP spikes were started on $\geq 1$ IOP lowering drop.

Overall, there was no difference in average RNFL thickness in treated versus untreated eyes $(80.4 \pm 15.5$ $\mu \mathrm{m}$ and $82.6 \pm 15.8 \mu \mathrm{m}$, respectively; $P=0.33$ ). The mean RNFL thicknesses between treatment and control arms stratified by diagnosis are summarized in Figure 3. The mean RNFL thicknesses between treatment and control arms stratified by the degree of IOP elevation are summarized in Figure 4.

\section{Prospective group}

Twenty-one eyes met the inclusion criteria. Sixty-five percent were male with the majority being Caucasian (71\%) and Hispanic (24\%). Seventy-one percent had a past ocular history of glaucoma made up primarily of primary open-angle glaucoma and glaucoma suspect, except for one patient with neovascular glaucoma. Eighty percent of the patients were started on 1 or more IOP lowering drops. The mean time between the initial IOP spike and followup OCT imaging was 13 months ranging from 3 to 50 months (median of 7 months). The mean IOP before the 
Average IOP elevation vs. diagnosis

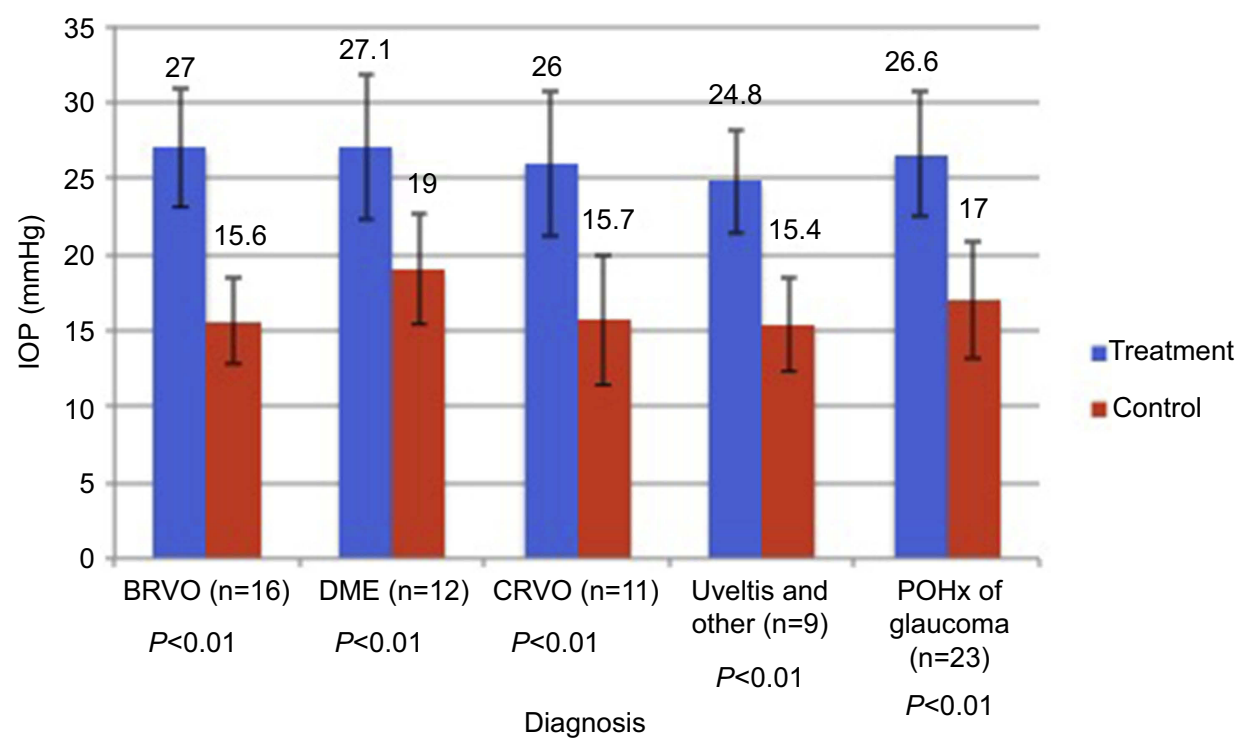

Figure 2 Above is a summary of the mean IOP between treatment and control arms stratified by diagnosis at the time of IOP spike in the cross-sectional group. P-value was $<0.05$ for each diagnosis. Other $=$ wAMD and CME.

Abbreviations: BRVO, branch retinal vein occlusion; DME, diabetic macular edema; CRVO, central retinal vein occlusion; wAMD, wet age-related macular degeneration; $\mathrm{CME}$, cystoid macular edema; IOP, intraocular pressure; $\mathrm{POHx}$, past ocular history.

Average RNFL thickness vs. diagnosis



Figure 3 Above is a summary of the mean RNFL thicknesses between treatment and control arms stratified by diagnosis. $P$-value was $>0.05$ for each diagnosis. Other $=$ wAMD and CME.

Abbreviations: BRVO, branch retinal vein occlusion; DME, diabetic macular edema; CRVO, central retinal vein occlusion; wAMD, wet age-related macular degeneration; CME, cystoid macular edema; POHx, past ocular history; RNFL, retinal nerve fiber layer.

DEX implant was $14.9 \pm 3.8 \mathrm{mmHg}$, while the mean IOP at the time of the IOP spike was $26.2 \pm 4.4 \mathrm{mmHg}(P<0.01)$. However, mean IOP had significantly decreased $4-8$ weeks after the IOP spike to $16.5 \pm 3.8 \mathrm{mmHg}(P<0.01)$. Overall, there was no statistically significant difference in the mean RNFL thicknesses before and after the IOP 
Avg. RNFL thickness vs. degree of IOP elevation

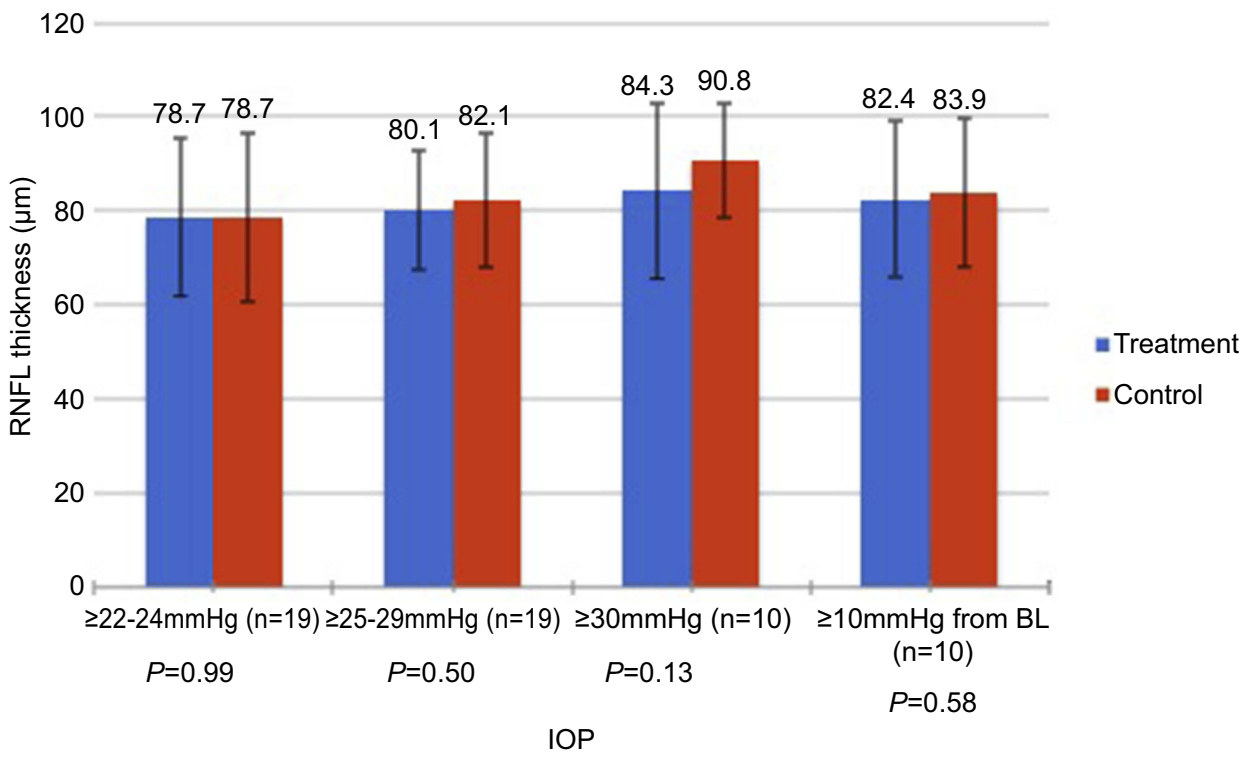

Figure 4 Above summarizes the mean RNFL differences between treatment and control arms stratified by the magnitude of IOP elevation as well as those treated eyes with IOP spikes $\geq 10 \mathrm{mmHg}$ from baseline IOP. $P$-value was $>0.05$ for each group.

Abbreviations: IOP, intraocular pressure; RNFL, retinal nerve fiber layer; BL, baseline; Avg, average.

spikes $(78.0 \pm 14.8 \mu \mathrm{m}$ and $75.6 \pm 13.6 \mu \mathrm{m}$, respectively; $P=0.13)$.

\section{Discussion}

Temporary IOP spikes related to DEX implantation for the treatment of macular edema have been noted as a common adverse effect in various studies. ${ }^{7,9,16-18}$ As many as onethird of eyes injected with DEX implant have been shown to experience this transient IOP spike with IOP control typically accomplished with topical IOP lowering drops. ${ }^{11,12}$ In addition, this risk of IOP increase appears to peak at 2 months but does not appear to incur cumulative IOP elevation with subsequent DEX implants. ${ }^{7,11,16}$ Given the substantial risk for IOP elevations, DEX implant is contraindicated for the treatment of macular edema in patients with glaucoma with a $\mathrm{CDR} \geq 0.8$. While efficacy and safety profiles have been established for DEX implant, no study to the best of our knowledge has been performed using OCT data to evaluate the RNFL thickness in patients who experience IOP spikes after treatment with DEX implant.

In the cross-sectional group, IOP spikes $\geq 22 \mathrm{mmHg}$ after DEX implantation demonstrated no significant difference in the average RNFL thickness when compared to the contralateral untreated eye regardless of treatment diagnosis, magnitude of IOP spike, or history of glaucoma. Of note, there was a significantly higher IOP in the treated eye when compared to the untreated contralateral eye at the time of IOP spike with both eyes having similar CCTs. Although a decrease in IOP 4-8 weeks after the IOP spike in the treated eye did not reach statistical significance in the cross-sectional group, the IOP did trend downward with none of the patients requiring incisional glaucoma surgery. This suggests that IOP spikes can be safely managed with topical IOP lowering drops until the steroid-induced IOP elevation resolves. The prospective group was also found to have no statistically significant difference in average RNFL thicknesses when comparing measurements before the IOP spike and at least 3 months after the IOP spike. IOP was significantly elevated above baseline IOP at the time of the IOP spike and was significantly lower 4-8 weeks after the IOP spike. Eighty percent of the patients in the prospective group received 1 or more IOP lowering drops to the lower IOP with none of them requiring incisional glaucoma surgery, demonstrating again that topical IOP lowering drops are effective and safe to use in those patients with steroid responses.

Glaucomatous optic neuropathy related to elevated IOP is postulated to occur at the microscopic level due to the inhibition of retrograde transport of essential trophic factors to the retinal ganglion cells (RGC). ${ }^{13,19}$ This disruption in axon transport subsequently leads to RGC death and glaucoma progression. Progression despite normal IOP, as seen with normal tension glaucoma, indicates that the process, however, is much more complex and is 
likely multifactorial. That being said, IOP is currently the only modifiable risk factor used to manage glaucoma. ${ }^{14}$

The standard of care for monitoring glaucoma progression is perimetric testing and OCT RNFL analysis. $^{20,21}$ Visual fields are an integral subjective analysis of functional visual loss related to glaucomatous damage but have been shown to have much intertest variation and issues with reproducibility. ${ }^{22}$ In addition, many patients treated with intravitreal steroids have impaired retinal function from their underlying disease, especially with RVO, making interpretation of perimetric testing difficult and subject to confounding visual field defects. While OCT RNFL analysis may not be associated with a functional visual field defect, its utility in demonstrating reproducible objective data with regards to the structure of the optic disc and thickness of the RNFL is crucial in monitoring for optic nerve damage and progression. ${ }^{23-25}$ It is for these reasons that OCT RNFL analysis was used in this study.

While the precise mechanism of steroid-induced IOP elevation is unknown, it is thought to be related to increased resistance to aqueous outflow through the trabecular meshwork similar to primary open angle glaucoma (POAG). ${ }^{26,27}$ However studies have shown that most temporary IOP spikes related to steroids can be successfully treated with topical IOP lowering agents alone. ${ }^{11,28,29}$ This was further demonstrated in our study with the majority of patients being started on 1 or more IOP lowering agents without evidence of RNFL thinning later or compared to the contralateral untreated eye. In addition, a significant number of patients in this study had a past ocular history of glaucoma, which shows that temporary IOP spikes even in this patient population can be safely managed on topical IOP lowering drops alone.

The primary weakness of this study is that RNFL thickness was not measured before initiation of DEX implant in the treatment nor control eye in the cross-sectional group. Therefore, whether there was a difference in RNFL thicknesses prior to DEX injection between either eye in each patient remains unknown. However, studies have shown that in normal adults inter-eye optic nerve cup to disc ratio is similar. ${ }^{30}$ Other studies have shown inter-ocular differences in average RNFL thicknesses; however, the actual degree of asymmetry is quoted as little as $0.3 \mu \mathrm{m} .{ }^{31}$ As far as the accuracy and precision of the RNFL thickness measurements, OCT offers good reliability and reproducibility. ${ }^{8,23-25}$ The prospective group offers a more reliable assessment of the RNFL of treated eyes as measurements were taken before and after the IOP elevation. Ideally, a series of 2 or more follow-up OCT RNFL studies would be performed and recorded to confirm or deny RNFL changes, however given the retrospective nature of this study that data was not available.

In conclusion, we demonstrate that temporary elevation of IOP after DEX implantation when treated with IOP lowering drops does not appear to cause a meaningful change in RNFL thickness, regardless of etiology or magnitude of IOP increase. Topical IOP lowering drops seem to be adequate in the management of temporary IOP spikes to prevent RNFL damage even in those patients with a past ocular history of glaucoma. Because of the small sample size, these findings must be interpreted with caution until larger future prospective studies are performed.

\section{Acknowledgments}

An abstract of this paper was presented at the annual Association of Research in Vision and Ophthalmology Conference (May 1, 2018) as a poster presentation with interim findings. The poster's abstract was published in "Poster Abstracts" in the Investigative Ophthalmology \& Visual Science Journal (ARVO journal):2018;59:2705. An abstract was also presented at the following meetings: Alamo Day annual meeting, San Antonio, TX, USA, April 7, 2018; Third Coast Retina Club, Austin, TX, USA, April 14, 2018; The Club Jules Gonin annual meeting; July 11-14, 2018; Jersey, Channel Islands (GB); and The American Academy of Ophthalmology annual meeting, Retina Subspecialty Day, 2018; Chicago, IL, USA.

Journal fees were funded by Allergan plc, Dublin, Ireland, through a publication grant. Otherwise, there was no other financial support.

\section{Disclosure}

Kendall Wannamaker, Sarah Kenny, Rishi Das, Aaron Mendlovitz, Jordan M Comstock, Edward R Chu, Sepehr Bahadorani, Nathan J Gresores, Kinley D Beck, Chelsey J Krambeer, Daniel S Kermany, Daniel P Nolan, and JeongHyeon Sohn report no conflicts of interest in this work. Roberto Diaz-Rohena reports personal fees from Allergan, outside the submitted work. Michael Singer reports grants from Allergan, during the conduct of the study, has been a consultant for Allergan, Genentech, Regeneron, Santen, Clearside Aerpio, and Alimera Speakers Bureau, and has received research support from Allergan, Genentech, Regeneron, Ampio, Optos, Aerpio, Allegro, Diachii, and Clearside. The authors report no other conflicts of interest in this work. 


\section{References}

1. Sarao V, Veritti D, Boscia F, Lanzetta P. Intravitreal steroids for the treatment of retinal diseases. The Scientific World Journal. 2014;2014:989501. doi:10.1155/2014/989501

2. Kang BS, Chung EY, Yun YP, et al. Inhibitory effects of anti-inflammatory drugs on interleukin-6 bioactivity. Biol Pharm Bull. 2001;24:701-703. doi:10.1248/bpb.24.701

3. Wilson CA, Berkowitz BA, Sato Y, Ando N, Handa JT, de Juan E Jr. Treatment with intravitreal steroid reduces blood-retinal barrier breakdown due to retinal photocoagulation. Arch Ophthalmol. 1992;110:1155-1159.

4. Fischer S, Renz D, Schaper W, Karliczek GF. In vitro effects of dexamethasone on hypoxia-induced hyperpermeability and expression of vascular endothelial growth factor. Eur $J$ Pharmacol. 2001;411:231-243.

5. Haghjou N, Soheilian M, Abdekhodaie MJ. Sustained release intraocular drug delivery devices for treatment of uveitis. J Ophthalmic Vis Res. 2011;6:317-329.

6. Chang-Lin JE, Attar M, Acheampong AA, et al. Pharmacokinetics and pharmacodynamics of a sustained-release dexamethasone intravitreal implant. Invest Ophthalmol Vis Sci. 2011;52:80-86. doi:10.1167/iovs.10-5285

7. Haller JA, Bandello F, Belfort R Jr., et al. Randomized, sham-controlled trial of dexamethasone intravitreal implant in patients with macular edema due to retinal vein occlusion. Ophthalmology. 2010;117(1134-46):e3. doi:10.1016/j.ophtha.2010.03.032

8. Lowder C, Belfort R Jr., Lightman S, et al. Dexamethasone intravitreal implant for noninfectious intermediate or posterior uveitis. Arch Ophthalmol. 2011;129:545-553. doi:10.1001/archophthalmol.2010. 339

9. Li X, Wang N, Liang X, et al. Safety and efficacy of dexamethasone intravitreal implant for treatment of macular edema secondary to retinal vein occlusion in Chinese patients: randomized, sham-controlled, multicenter study. Graefe's Arch Clin Exp Ophthalmol. 2018;256:59-69. doi:10.1007/s00417-017-3831-6

10. Kuppermann BD, Goldstein M, Maturi RK, et al. Dexamethasone intravitreal implant as adjunctive therapy to ranibizumab in neovascular age-related macular degeneration: a multicenter randomized controlled trial. Ophthalmologica $J$ Int d'ophtalmologie Int J Ophthalmol Z fur Augenheilkunde. 2015;234:40-54. doi:10.11 $59 / 000381865$

11. Capone A Jr., Singer MA, Dodwell DG, et al. Efficacy and safety of two or more dexamethasone intravitreal implant injections for treatment of macular edema related to retinal vein occlusion (Shasta study). Retina. 2014;34:342-351. doi:10.1097/IAE.0b013e31829 $7 \mathrm{f} 842$

12. Boyer DS, Yoon YH, Belfort R Jr., et al. Three-year, randomized, sham-controlled trial of dexamethasone intravitreal implant in patients with diabetic macular edema. Ophthalmology. 2014;121:1904-1914. doi:10.1016/j.ophtha.2014.04.024

13. Quigley HA, McKinnon SJ, Zack DJ, et al. Retrograde axonal transport of BDNF in retinal ganglion cells is blocked by acute IOP elevation in rats. Invest Ophthalmol Vis Sci. 2000;41:3460-3466.

14. Boland MV, Ervin AM, Friedman DS, et al. Comparative effectiveness of treatments for open-angle glaucoma: a systematic review for the U.S. preventive services task force. Ann Int Med. 2013;158:271-279. doi:10.7326/0003-4819-158-4-201302190-00008

15. Bucolo C, Gozzo L, Longo L, Mansueto S, Vitale DC, Drago F. Long-term efficacy and safety profile of multiple injections of intravitreal dexamethasone implant to manage diabetic macular edema: a systematic review of real-world studies. J Pharmacol Sci. 2018;138:219-232. doi:10.1016/j.jphs.2018.11.001
16. Kuppermann BD, Blumenkranz MS, Haller JA, et al. Randomized controlled study of an intravitreous dexamethasone drug delivery system in patients with persistent macular edema. Arch Ophthalmol. 2007;125:309-317. doi:10.1001/archopht.125.3.309

17. Eter N, Mohr A, Wachtlin J, Feltgen N, Shirlaw A, Leaback R. Dexamethasone intravitreal implant in retinal vein occlusion: real-life data from a prospective, multicenter clinical trial. Graefe's Arch Clin Exp Ophthalmol. 2017;255:77-87. doi:10.1007/s00417016-3431-x

18. Korobelnik JF, Kodjikian L, Delcourt C, et al. Two-year, prospective, multicenter study of the use of dexamethasone intravitreal implant for treatment of macular edema secondary to retinal vein occlusion in the clinical setting in France. Graefe's Arch Clin Exp Ophthalmol. 2016;254:2307-2318. doi:10.1007/s00417-016-3394-y

19. Burgoyne CF, Downs JC, Bellezza AJ, Suh JK, Hart RT. The optic nerve head as a biomechanical structure: a new paradigm for understanding the role of IOP-related stress and strain in the pathophysiology of glaucomatous optic nerve head damage. Prog Retin Eye Res. 2005;24:39-73. doi:10.1016/j.preteyeres.2004.06.001

20. Kersey T, Clement CI, Bloom P, Cordeiro MF. New trends in glaucoma risk, diagnosis \& management. Indian $J$ Med Res. 2013;137:659-668.

21. De Moraes CG, Liebmann JM, Levin LA. Detection and measurement of clinically meaningful visual field progression in clinical trials for glaucoma. Prog Retin Eye Res. 2017;56:107-147. doi:10.1016/j. preteyeres.2016.10.001

22. Phu J, Khuu SK, Yapp M, Assaad N, Hennessy MP, Kalloniatis M. The value of visual field testing in the era of advanced imaging: clinical and psychophysical perspectives. Clin Exp Optometry. 2017;100:313-332. doi:10.1111/cxo.2017.100.issue-4

23. Leung CK. Diagnosing glaucoma progression with optical coherence tomography. Curr Opin Ophthalmol. 2014;25:104-111. doi:10.1097/ ICU.0000000000000024

24. Leung CK, Cheung CY, Weinreb RN, et al. Retinal nerve fiber layer imaging with spectral-domain optical coherence tomography: a variability and diagnostic performance study. Ophthalmology. 2009;116:1257-63,63 e1-e2. doi:10.1016/j.ophtha.2009.04.013

25. Leung CK, Lam S, Weinreb RN, et al. Retinal nerve fiber layer imaging with spectral-domain optical coherence tomography: analysis of the retinal nerve fiber layer map for glaucoma detection. Ophthalmology. 2010;117:1684-1691. doi:10.1016/j.ophtha.2010.01.026

26. Clark AF, Wordinger RJ. The role of steroids in outflow resistance. Exp Eye Res. 2009;88:752-759. doi:10.1016/j.exer.2008.10.004

27. Weinreb RN, Aung T, Medeiros FA. The pathophysiology and treatment of glaucoma: a review. Jama. 2014;311:1901-1911. doi:10.1001/jama.2014.3192

28. Kramar M, Vu L, Whitson JT, He YG. The effect of intravitreal triamcinolone on intraocular pressure. Curr Med Res Opin. 2007;23:1253-1258. doi:10.1185/030079907X187946

29. Unsal E, Eltutar K, Sultan P, Gungel H. The efficiency of intravitreal dexamethasone implants in the treatment of macular edema secondary to retinal vein occlusion. $J$ Ocul Pharmacol Ther. 2015;31:350-356. doi:10.1089/jop.2014.0141

30. Li H, Healey PR, Tariq YM, Teber E, Mitchell P. Symmetry of optic nerve head parameters measured by the heidelberg retina tomograph 3 in healthy eyes: the blue mountains eye study. Am J Ophthalmol. 2013;155(518-23):e1. doi:10.1016/j.ajo.2012.09.019

31. Dalgliesh JD, Tariq YM, Burlutsky G, Mitchell P. Symmetry of retinal parameters measured by spectral-domain OCT in normal young adults. $J$ Glaucoma. 2015;24:20-24. doi:10.1097/ IJG.0b013e318287ac2f 


\section{Publish your work in this journal}

Clinical Ophthalmology is an international, peer-reviewed journal covering all subspecialties within ophthalmology. Key topics include: Optometry; Visual science; Pharmacology and drug therapy in eye diseases; Basic Sciences; Primary and Secondary eye care; Patient Safety and Quality of Care Improvements. This journal is indexed on PubMed
Central and CAS, and is the official journal of The Society of Clinical Ophthalmology (SCO). The manuscript management system is completely online and includes a very quick and fair peer-review system, which is all easy to use. Visit http://www.dovepress.com testimonials.php to read real quotes from published authors. 